\title{
Advances in Inertial Measurement Technology for Marine Motion Control
}

\author{
MATHIAS HÅNDLYKKEN* \\ Keywords: Attitude Sensor, Roll Control, Pitch Control, Marine Auto Pilot
}

This paper describes the function of an inertial "strap down" attitude sensor based on solid state Coriolis force rate gyros, accelerometers and magnetic sensor. Performance is analyzed taking into account the typical excitations in attitude and linear motion seen in marine applications. The use is for control of fast crafts, ROV \& AUV heading, roll, pitch and heave control. The influence on performance given by utilization of external information from velocity log and more accurate heading devices is also analyzed. Typical performance of this low cost type of technology is shown.

\section{Introduction}

The technology for inertial measurement of attitude has primarily been developed for avionics. The niches of civilian maritime applications have been covered by use of the same type of technology. The result of this is a mismatch between product performance and customer requirements in the latter applications. It is now seen as a trend that more advanced control technology is entering the maritime niche when talking about vehicle control.

In "high end" military maritime applications like gun fire control systems the required technology is matching with the laser gyro technology/dynamically tuned mechanical gyro technology initially developed for avionics.

For applications such as roll, pitch, heave stabilization of ships and subsea vehicles this is often "overkill" on accuracy. The customers main requirement is often to have a low cost unit that can operate over extended time periods without need for maintenance. The niche of maritime applications for inertial technology is not large enough to efficiently allow vertical integration within a company to include the development and production of the prime inertial sensors. The gyro and accelerometer technology should be based on spin-off from higher volume applications to achieve a cost efficient solution.

Two attractive sources for basic sensor technology for maritime applications can be mentioned: The first is technology for low cost short range inertially guided missiles, the second is technology for the "high end" automobile market. In the last-mentioned case inertial sensors are used for the latest anti-skid-systems and active suspension systems.

Seatex AS has developed a product (Seatex Motion Reference Unit, later called MRU) based on gyro technology developed for the automobile industry. In this paper

Received 20 August 1995

*Seatex As, Pirsenteret 7005, Trondheim, Norway. Fax: (47)-73515020. Phone: (47) 73545500.

Presented at the 3rd IFAC Workshop on Control Applications in Marine Systems, May 1995, Trondheim, Norway (CAMS '95). 
it is investigated how low accuracy "automobile" sensors can be used in maritime applications by utilization of extensive error modeling.

\section{Performance Requirements}

Typical needed data for an attitude sensor for maritime fast craft and ROV control applications:

$\begin{array}{ll}\text { Static error } & :<0 \cdot 2 \mathrm{Deg} \\ \text { Dynamic error under all condo. } & :<0 \cdot 3 \mathrm{Deg} \\ \text { Resolution } & :>0 \cdot 05 \mathrm{Deg} \\ \text { Angular vel resolution } & :>0.05 \mathrm{Deg} / \mathrm{s} \\ \text { Maximum range } & :>50 \mathrm{Deg} / \mathrm{s} \\ \text { Sample rate } & :>20 \mathrm{~Hz} \\ \text { Signal bandwidth } & :>10 \mathrm{~Hz}\end{array}$

Even if the typical rolloff frequency is $1 \mathrm{~Hz}$ in the control loops of the vehicle there is a need for low phase delay in the roll/pitch sensors at the rolloff frequency, thus requiring an order of magnitude higher sensor bandwidth.

The need for high resolution is given by the need to minimize the operation of thrusters/fins/rudders during operation.

A fundamental difference between avionics use and maritime use is found in the needed vehicle acceleration insensitivity. In maritime applications are the dominating accelerations high-frequency ocean-wave induced motion, and not accelerations created by maneuvering of the craft itself.

This means that the direction of the total measured acceleration (including g) can be better utilized to find the down-direction than for aircrafts. This correspondingly means less requirements to the rate gyros for maritime use and puts less requirements to the rate gyro technology to keep accurate attitude over long time.

\section{3. "Strap Down" Attitude Computation}

An overview of the chosen implementation is shown on Figure 1. The system has rate gyroscopes and accelerometers directly fixed to the sensor body without using a gimbal system. This is called a "strap down" implementation of the inertial attitude sensor and means no complex mechanics or moving parts. Symbols on Figure 1:

$\boldsymbol{\omega}_{\mathrm{m}}{ }^{\mathrm{s}}, \boldsymbol{a}_{\mathrm{m}}{ }^{\mathrm{s}}, \boldsymbol{b}_{\mathrm{m}}{ }^{\mathrm{s}}$ Measured angular velocity, acceleration magnetic field in nonorthogonal sensor frame

$\omega^{\mathrm{b}}, \boldsymbol{a}^{\mathrm{b}}, \boldsymbol{b}^{\mathrm{b}} \quad$ A priori error-compensated measurements in body frame

$\hat{\boldsymbol{\omega}}^{\mathrm{b}} \quad$ Estimated angular velocity in body frame

$C_{b}^{\mathrm{g}} \quad$ Rotation matrix from body to navigation-frame

$\alpha^{\mathrm{b}} \quad$ Rate gyro error parameters in body frame, estimated at run-time

$e^{\mathrm{g}} \quad$ Instantaneous attitude error in navigation frame

Again referring to Figure 1, the upper blocks 1, 4, 7 and 10 contain the fast angular rate processing. Block 1 is the sensor for the angular rate vector based on Coriolis force vibratory gyroscopes. Block 4 is an "a priori" angular error correction where parameters are determined during factory calibration. Block 7 is an angular rate error correction where parameters are determined during operation of the system. Block 10 is the solution of the basic angular rate to orientation differential equation. The output from 


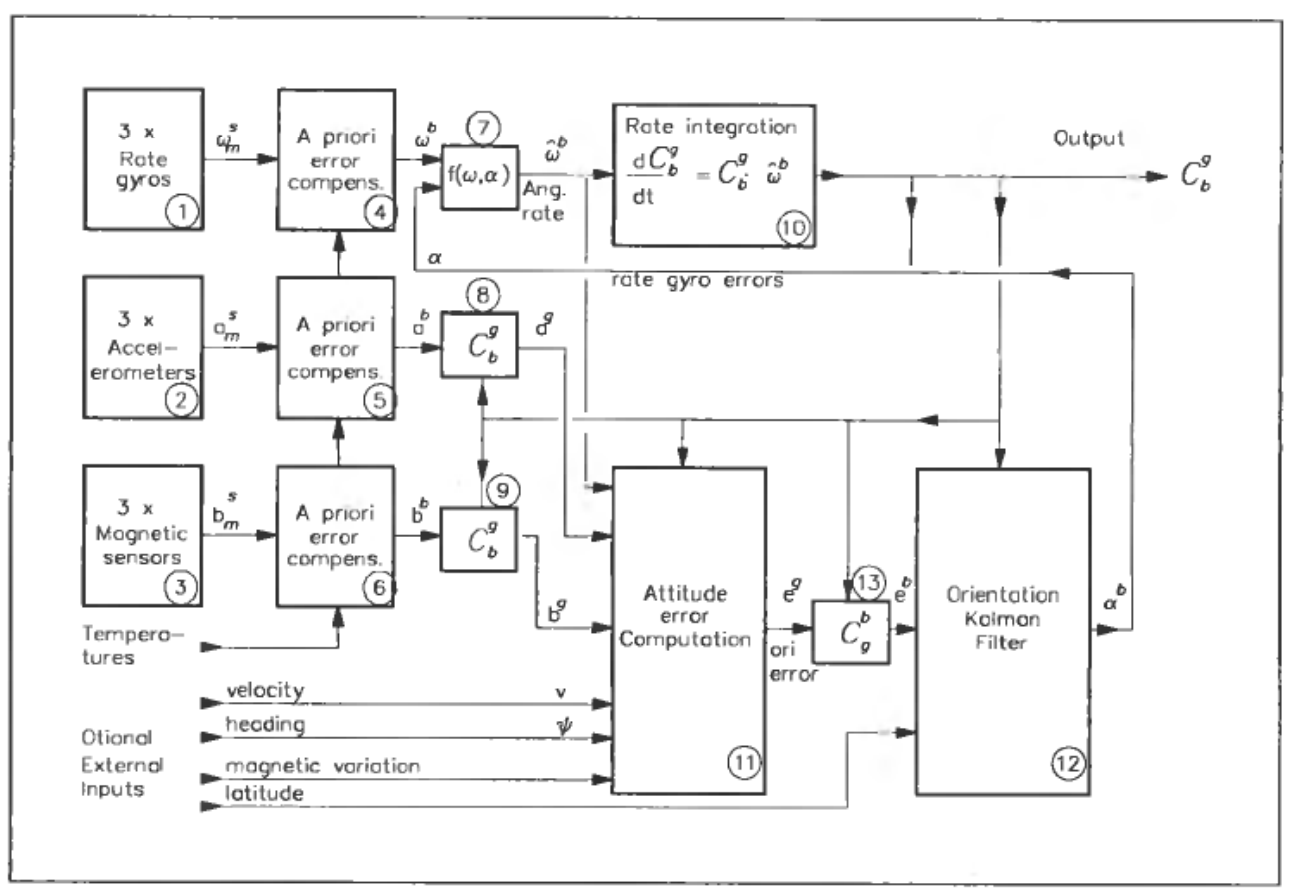

Figure 1. Overview of attitude part of the "strap down" implementation in the Seatex MRU.

the system is a rotation matrix $\boldsymbol{C}_{b}^{g}$ between body coordinates and navigation coordinates (in reality compactly represented as 3 Euler angles or as symmetric Euler parameters).

Block 2 is measuring the acceleration vector. A priori error compensation is performed as in block 4 for angular rate. Block 8 transforms acceleration from body to navigational frame compensating for the so-called sculling effect, i.e. rotation during the acceleration measurement cycle. Block 3 measures the terrestrial magnetic field vector with error corrections in block 6 and transformation to navigational frame including de-sculling in block 9 as for the acceleration. The operation in block 6 is extended compared to block 4 and 5 because also vehicle magnetic parameters can be included in the error compensation. Blocks 3,6,9 are optionally used when reliable external heading information is not available. Block 11 computes an instantaneous attitude error vector based on the best computed direction of gravity. Included here is lever arm correction to the vehicle CG, compensation for centripetal and tangential accelerations from vehicle maneuvering, anti-aliasing filtering for the output error vector $\boldsymbol{e}^{\mathrm{g}}$.

Block 12 is a Kalman filter computing rate gyro errors in the body-frame. This filter operates on an error model for each of the 3 rate gyros. Earth rate compensation is also performed in this filter.

Generally the implementation of the "strap-down" algorithms herein is possible to make more "clean" and simple than what is possible in an in-flight alignment inertial navigation system. This is, given by lower accuracy requirements combined with some recent algorithm improvements.

Some of the blocks mentioned above will be analyzed in more details below. We will particularly pay attention to the angular rate processing. blocks $1,4,10$ and 11 . 


\section{Rate gyros based on the Coriolis force}

The MRU contains 3 rate gyros placed in block 1 on Figure 1. For maritime applications long lifetime is wanted since the system usually will be in operation during the whole lifetime of the ship. Rate gyros based on vibratory linear motion have some attractive properties:

* True solid state function, no wear-out

* Low kinematic rectification

* High resolution

* Low g sensitivity

* Low vibration sensitivity (not valid for all CFG-designs)

The second property means that oscillatory motions (given by ocean waves) will provide small accumulating angular errors. Other less good properties are:

* moderate bias stability

* moderate scale factor accuracy

The last properties have to be overcome by error modeling, the requirements are also moderate in these applications. Typically is a scale factor stability of 0.1 to $0.5 \%$ sufficient for the 2 horizontal rotation axes, and $0.1 \%$ for the vertical rotation axis. To avoid a non-symmetric design and to allow for mounting in all orientations of the system on the vessel all 3 axis directions should have the same performance as required for the vertical rotation axis. Similar requirements for avionics applications is around 1 order of magnitude higher. The requirements for bias long term stability is very moderate. However short term stability in the area of 5 minutes must be down in the area of $0.001-0.005 \mathrm{Deg} / \mathrm{s}$. This is given by that optimal times for erection of attitude towards measured direction of acceleration is in the order of 5 minutes, and integrated rate during this time must be well below the accuracy limits in attitude. This primarily means that good temperature compensation and low bias sensitivity for vibrations and mechanical shocks is needed. Also internal effects leading to sudden bias or scale factor changes must be small.

The chosen Coriolis gyro design is based on the use of a vibrating cylinder. This type of sensor has been analyzed by Burdess (1986), Fox (1988) and others. There are also numerous other Coriolis force gyro designs under development and some in production. Not all potential manufacturers have focused enough on the advanced underlaying material technology problems and are ending up with poor designs, others have ended up with extremely complex nearly non-manufacturable designs. It is however now reasonable to believe that this type of technology will develop by the push from the automobile industry to a level where it is almost unbeatable in price and reliability by other (less production-optimized) technology when we are talking about the lower performance range of rate gyros. Referring to Fig. 2 is the generated corriolis acceleration given as:

$$
a_{c}=-2 \cdot \omega \times \alpha_{0} \cdot \sin \left(\omega_{0} \cdot t\right)
$$

where $\omega_{0}$ is the vibration frequency and $\mathrm{a}_{0}$ is the amplitude. Typical values for $\omega_{0}$ is $10^{5}$ and $\mathrm{a}_{0}=1 \mu \mathrm{m}$. With a resolution $/ \mathrm{noise}$ level at $\omega=10^{-4} \mathrm{rad} / \mathrm{s}(0.0057 \mathrm{Deg} / \mathrm{s})$ this means from (1) a Coriolis force amplitude of $2 \cdot 10^{-5} \mathrm{~m} / \mathrm{s}^{2}$.

There are no fundamental electronic noise problems with the measurements of these levels of accelerations (not true for micro-machined Coriolis force rate gyro designs). Environmental sensitivity concerning vibrations has occassionally been a claim against 


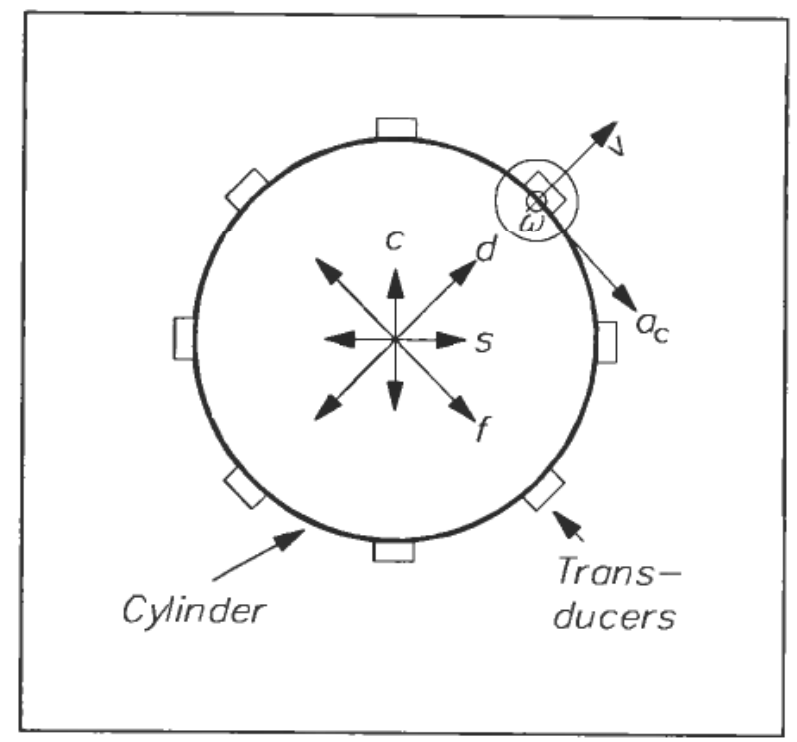

Figure 2. Coriolis Force rate Gyro function.

d Dive axis direction

f Feedback, measurement of drive axis

s Sensing axis

c Compensation axis

$\omega$ Rotation rate in an axis normal to the paper plane

$\mathrm{v}$ Linear velocity set up by driven oscillation

$\mathrm{a}_{\mathrm{c}}$ Coriolis acceleration set up by the rotation rate $\omega$

this rate gyro technology, this can however easily be overcome by good design. To avoid environmental vibration sensitivity 3 methods are used in the same design:

* Use of a high drive frequency, the vehicle has lower noise at these frequencies

* Damping of external accelerations (typically $60 \mathrm{~dB}$ ) by a symmetric design of the sensor cylinder. A cylinder design is superior to most other mechanizations. (The effects of mass unbalance of cylinders has been studied by Fox 1994)

* Additional damping by mechanical filter ( $40 \mathrm{~dB}$ is easily achieved). This can be a very simple spring/mass device when $\omega_{0}$ is in the order of $10^{5} \mathrm{rad} / \mathrm{s}$

It is easy to get total environmental suppression (even at the drive frequency of several $\mathrm{m} / \mathrm{s}^{2}$ ) with this design, since the environment amplitude will be damped typically $10^{6}$. The sensor is implemented as a force feedback system. The d-axis on Figure 2 is used for excitation, the f-axis to measure the drive amplitude for control of the excitation. A measurement feedback loop is controlling an excitation along the c-axis which is cancelling the vibration set up along axis s given by the Coriolis acceleration $\mathrm{a}_{\mathrm{c}}$ which has to be measured. The drive signal to the $\mathrm{c}$-axis is thus the final output from the rate sensor.

This rate gyro feedback loop is in itself a complex matter if high performance is needed. To achieve high bandwidth and high accuracy the vibratory dynamics of the rate gyro is modeled and used in a state estimator/controller for the rate gyro. This loop operates in the drive frequency band and needs a dynamic signal range of $90 \mathrm{dR}$. 


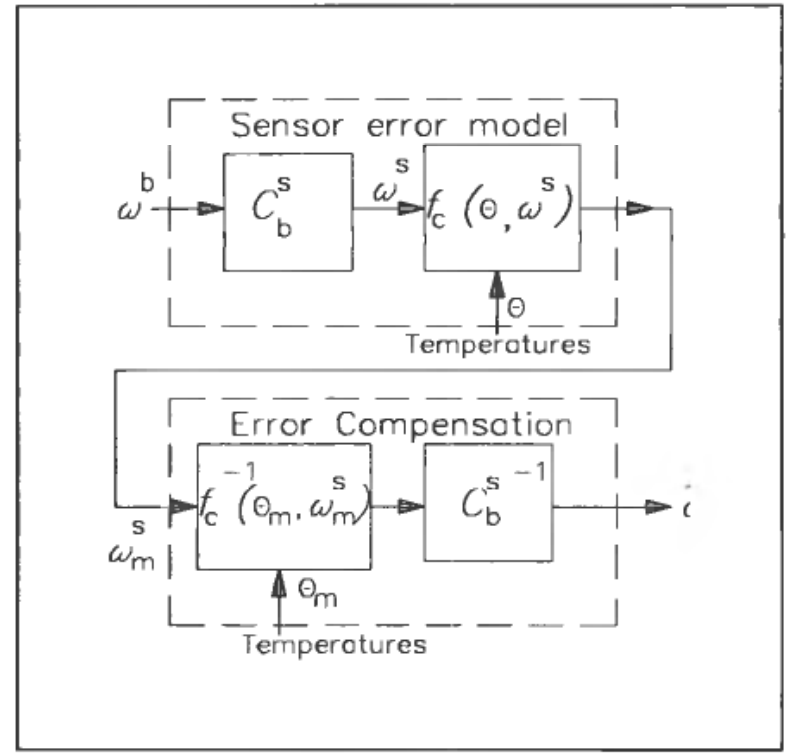

Figure 3. Rate channel a priori error compensation.

Used symbols on Figure 3:

$\boldsymbol{C}_{\mathrm{b}}^{\mathrm{s}} \quad$ Transformation matrix from body frame to non-orthogonal sensor frame $\mathbf{f}_{\mathrm{c}}\left(\theta, \omega^{\mathrm{s}}\right)$ Sensor measurement function including sensor temperature sensitivity

\section{Rate gyro a priori error corrections}

This is performed on block 4 on Figure 1. The used method is shown on Fig. 3. Similar schemes are used for the accelerometers and the magnetic sensor. The overall philosophy is to minimize number of parameters to be observed during normal operation of the MRU and maximize number of parameters to be determined during calibration.

This is because high observability in normal run cannot be assured, and hence minimum performance cannot be guaranteed.

The axis directions are not assumed to be a function of temperature. Hence we first have a linear transformation from orthogonal body frame to a non-orthogonal sensor frame $\boldsymbol{C}_{\mathrm{b}}^{\mathrm{s}} . \boldsymbol{C}_{\mathrm{b}}^{\mathrm{s}}$ is close to ortho-normal since deviations are small mounting errors of the sensors in the sensor house in addition to internal sensor direction errors. $\boldsymbol{C}_{\mathrm{b}}^{\mathrm{s}}$ is hence well behaved at inversion. The same is true for fc which is monotonic and actually close to a straight line.

Acceleration-dependency is not modeled in $\mathrm{fc}$ since this dependency is marginal for the chosen type of gyroscopes. The computation is hence:

$$
\omega^{\mathrm{b}}=\left(\boldsymbol{C}_{\mathrm{b}}^{\mathrm{s}}\right)^{-1} \mathbf{f}_{\mathrm{c}}^{-1}\left(\theta_{\mathrm{m}}, \omega_{\mathrm{m}}^{\mathrm{s}}\right)
$$

An important point is to ensure that there is small dependency on spatial/time gradients of temperature, i.e. representative temperature measurements must be available. This is achieved using high thermal conductivity internally, and having low power dissipation inside the sensor housing.

The function $\mathbf{f}_{\mathrm{c}}\left(\theta, \omega^{\mathrm{s}}\right)$ is for the angular rate set up with 2 . order polynomials for temperature dependencies, and 3 . order for sensor nonlinearity approximation. 
Linearity errors tend to be symmetric around zero rate for these types of sensors, hence a 3. order term is needed even for course modeling. Polynomials are computationally efficient in operation of the MRU (high throughput rate), and if low order is sufficient the well-known problem of "oscillation" between the target points is not present.

The function $\mathbf{f}_{\mathrm{c}}\left(\theta, \omega^{\mathrm{s}}\right)$ contains 24 parameters. Additionally the $\boldsymbol{C}_{\mathrm{b}}^{\mathrm{s}}$ contains 6 independent parameters after being normalized. Hence the angular rate channel contains 30 independent parameters to be observed during initial calibration/burn-in.

A systematic angular rate excitation under varying temperatures is needed to be able to safely determine all the above parameters.

A computerized method is used to guide the operator/collect data/estimate the parameters, during burn-in/calibration to ensure that all needed data is collected.

Parameter fitting is performed using a standard weighted chi-square method. Parameters are actually estimated in 2 steps:

(a) Let $\boldsymbol{C}_{\mathrm{b}}^{\mathrm{s}}=\mathbf{I}$, i.e. no cross-coupling assumed

(b) Estimate the 24 parameters of fc using the method LSQ1 (Press, 1988)

(c) Estimate the 6 parameters of $\boldsymbol{C}_{\mathrm{b}}^{\mathrm{s}}$ pr axis (linear problem) using singular value decomposition, method LSQ2 (Press, 1988)

(d) Repeat the process a, b, c, check for change in residues

The residues provide important information on operator errors during this process, sensor errors/errors in electronics, and is used in the quality control for each tested MRU.

Since computing time is not a problem for this even with a slow Personal Computer not much effort was spent in finding a more sophisticated computer/time efficient estimation method for these parameters.

The found parameters are downloaded to the MRU without any hardware intervention, thus easing the calibration immediately after data is collected. This also means that recalibration of the MRU at a later date is a simple operation.

\section{Angular rate integration}

The fundamental equation from mechanical dynamics (3) is solved in block 10 on Figure 1:

$$
\frac{d \boldsymbol{C}_{\mathrm{b}}^{\mathrm{g}}}{d t}=\boldsymbol{C}_{\mathrm{b}}^{\mathrm{g}} \cdot \Omega_{m}{ }^{b}
$$

where $\Omega_{m}{ }^{b}$ is the skew symmetric form of $\omega^{b}$. A solution to (3) can in principle be achieved directly by a series of expansion of the matrix exponential, however it is more practical to use other methods. One is to use an equivalent formulation to (3) based on symmetric Euler parameters (eq. 5 below), the last is used in the MRU:

$$
\frac{d \mathbf{q}}{d t}=\frac{1}{2}\left|\begin{array}{cccc}
0 & \omega 3 & -\omega 2 & \omega 1 \\
-\omega 3 & 0 & \omega 1 & \omega 2 \\
\omega 2 & -\omega 1 & 0 & \omega 3 \\
-\omega 1 & -\omega 2 & -\omega 3 & 0
\end{array}\right| \cdot \mathbf{q}=\mathbf{A}_{q} \cdot \mathbf{q}
$$

where 
q The 4 Euler parameters corresponding to $\boldsymbol{C}_{\mathrm{b}}^{\mathrm{g}}$

$\omega_{1}-\omega_{3}$ The 3 components of $\omega^{\mathrm{b}}$

The angular velocity $\omega^{\mathrm{b}}$ is in reality only available as angular rate increments $\Delta \theta^{\mathrm{b}}$ for each sample of data $n$ through block 1 and 4 on Figure 1:

$$
\Delta \boldsymbol{\theta}^{\mathrm{b}}=\int_{t_{n}}^{t_{n}+1} \boldsymbol{\omega}^{\mathrm{b}} \mathrm{dt}
$$

The rate gyro interface is doing a fast integration of the angular rates to avoid aliasing errors set up by high frequency mechanical vibrations. For commuting motion (i.e. when succession of incremental rotations from time $t_{n}$ can be interchanged for unchanged end orientation at $t_{n+1}$ ) the exact solution of 4 can be expressed as:

$$
\mathbf{q}\left(t_{n+1}\right)=\left[e \int_{t_{n}}^{t_{n}+1} \mathbf{A}_{q}(t) d t\right] \cdot \mathbf{q}\left(t_{n}\right)
$$

Since $\mathbf{A}_{\mathrm{q}}$ contains the angular rates, the integral of $\mathbf{A}_{\mathrm{q}}$ can be expressed as the similar matrix of components of $\Delta \theta^{\mathrm{b}}$. Hence the solution of (6) is:

$$
\mathbf{q}\left(t_{n+1}\right)=e^{\mathbf{A}_{q}(\Delta \theta b(n))} \cdot \mathbf{q}\left(t_{n}\right)
$$

(7) can be solved numerically by a series expansion of the matrix exponential. Typically 4 terms are needed. Additionally the quaternion $q$ must be normalized regularly to avoid build-up of numerical errors. To make the solution exact in the general motion case the $\Delta \boldsymbol{\theta}^{\mathrm{b}}$ in equation (7) must have a coning or non-commutivity correction added (Bortz 1973):

$$
\Delta \theta^{\mathrm{b}}(n)=\int_{t_{n}}^{t_{n} t^{\prime}} \omega^{b}(t) \cdot d t+\frac{1}{2} \int_{t_{n}}^{t_{n}+1} \operatorname{skew}\left(\int_{t_{n}}^{t} \omega^{b}(\tau) \cdot d \tau\right) \cdot \boldsymbol{\omega}^{b}(t) d t
$$

where skew() means skew symmetric form of (). The last term in (8) can be approximated in a number of ways. Some good approximations are methods E. to .H in Ignagni (1990) and Miller (1983). In traditional "strap down" INS implementations it is normal to run the deconing term at high sample rate (typically $1-2 \mathrm{kHz}$ ). To do this the sensor data processing in block 4 on Figure 1 has typically been bypassed given by too high computer time consumption for the de-coning-processing. This is messing up the computational scheme.

By using the modern deconing algorithms, combined with the relaxed requirements for accuracy needed in the MRU, high speed deconing is no longer needed. All data can be handled at a rate of $200 \mathrm{~Hz}$ for all processing.

For the MRU the most heavy coning motion is found when a small craft is turning to tack against the waves at high speed. Peak motion around $1 \mathrm{~Hz}$ and up to $0 \cdot 1$ radian amplitude can be worst case. At $200 \mathrm{~Hz}$ sample rate a satisfactory coning rejection is achieved even with a rudimentary deconing-algorithm-like approximating the last term of equation ( 8 ) by the $1 / 2$ cross-product between the last and the current integration of the angular rate over the sample interval. $\left(10^{-4}\right.$ in rejection, Ignagni (1990), deconing method A.)

Unbalance in propellers and machinery combined with strong local structural resonances can however provide amplitudes up to $0.01 \mathrm{rad}$ and frequency up to $10 \mathrm{~Hz}$. 
At these frequencies the new algorithms will achieve an order of magnitude improvement over traditional algorithms, Ignagni (1990). Providing a total coning rejection of $10^{-4}$ also at the machinery frequencies.

\section{Attitude error computation}

This process is performed by block 11 on Figure 1. The input acceleration in navigation frame $\mathbf{a}^{\mathrm{g}}$ will be exactly equal to gravity if the vehicle has no acceleration because of motion, and if there are no attitude errors present in the MRU. An attitude error in the MRU will result in measured horizontal components of acceleration in $\mathbf{a}^{\mathrm{g}}$ even if the vehicle is at rest.

The horizontal accelerations of $\mathbf{a}^{\mathrm{g}}$ can hence be used to compute the small attitude error as:

$$
\begin{aligned}
\Delta \varphi_{\text {North }} & \approx \frac{-\mathbf{a}_{\text {East }}{ }^{g}}{g} \\
\Delta \varphi_{\text {East }} & \approx \frac{-\mathbf{a}_{\text {North }}{ }^{g}}{g}
\end{aligned}
$$

The magnetic input to block 11 on Figure 1 is providing the expected terrestrial magnetic field in nav-frame:

$$
\Delta \varphi_{\text {Down }} \approx \tan ^{-1}\left(\frac{-\mathbf{b}_{\text {East }}{ }^{g}}{\mathbf{b}_{\text {North }}{ }^{g}}\right)-\varphi_{\text {Vari }}
$$

where $\varphi_{\text {Vari }}$ is the local magnetic variation. Alternatively the heading error in (10) is derived from available external heading. The 3 error angles constitute the 3 components of the error vector $\mathrm{e}^{\mathrm{g}}$ output from block 11.

Two conditions should be taken into account when using (9):

(a) Waves and fast maneuvers will provide large instantaneous error angles in (9) above, however, only the long time average of these will contribute to attitude errors seen in the output from the MRU.

(b) Long term turning of the vehicle (running in circle is the worst case), will create a centrifugal acceleration which makes an error in 9 even after averaging. Long term acceleration of the vehicle will create a similar effect. The MRU needs to erect attitude from acceleration in a few minutes to preserve accuracy. A single turn will hence influence attitude.

To minimize the influence of $(a)$ above a lever arm correction transforming the acceleration from the measurement point to the most silent location of the vehicle (normally ships CG) is useful:

$$
\mathbf{a}_{c o r r}{ }^{g}=\mathbf{a}^{g}+\mathbf{C}_{b}^{g}\left(\boldsymbol{\Omega}^{b 2}+\frac{d \boldsymbol{\Omega}^{b}}{d t}\right) \cdot \mathbf{p}_{c g}{ }^{b}
$$

where $\mathbf{p}_{c g}{ }^{b}$ is the fixed lever arm vector from measurement point to the vehicles CG.

To correct for $(b)$ above, utilization of external information about speed,velocity or vehicle acceleration can be useful. Most easily available is speed from a speedlog. A maneuvering correction term to $\mathbf{a}^{g}$ can now be subtracted:

$$
\mathbf{a}_{v}{ }^{g}=\mathbf{a}^{\mathrm{g}}+\mathbf{C}_{b}^{g}\left[\frac{d v}{d t}, \omega_{\text {Yuxis }}{ }^{b} \cdot v, 0\right]^{T}
$$


0
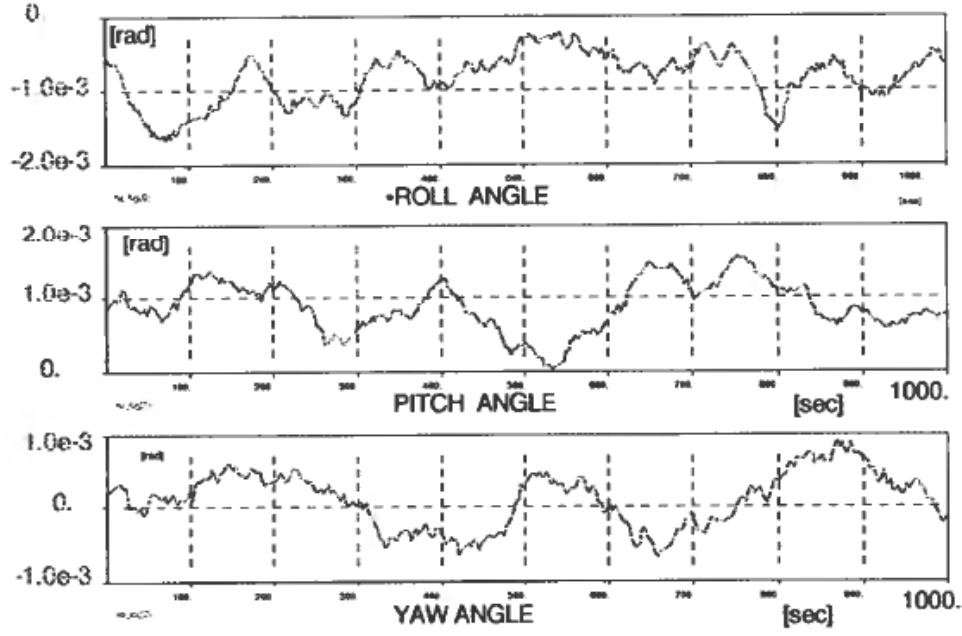

Figure 4. Intrinsic noise level in roll, pitch, yaw.

where $v$ is the vehicle forward speed and $\omega_{\text {Yaxis }}{ }^{b}$ is the angular velocity around the vehicle down (Y)-axis. For correctness (12) requires that vehicle heading and track-speed direction is the same. This is not quite an accurate assumption for all crafts. The accuracy in $\omega_{\text {Yaxis }}^{b}$ must also be good, which means that reasonable good heading must be available, or a good bias stability in $\omega_{\text {Yaxis }}{ }^{b}$ is needed. Experiments with 40 knots fast foil catamarans show that speed input is sufficient with the MRU for achieving the performance goals. An accurate method of correction is to utilize the GPS navigation system providing velocity and position, combined with acceleration data from the MRU in the nav-frame. The estimated vehicle acceleration bias will now represent the attitude error in the MRU. This is used in a new product from Seatex AS called Seapath ${ }^{\circledR}$ where the MRU is tightly integrated with GPS.

\section{Results}

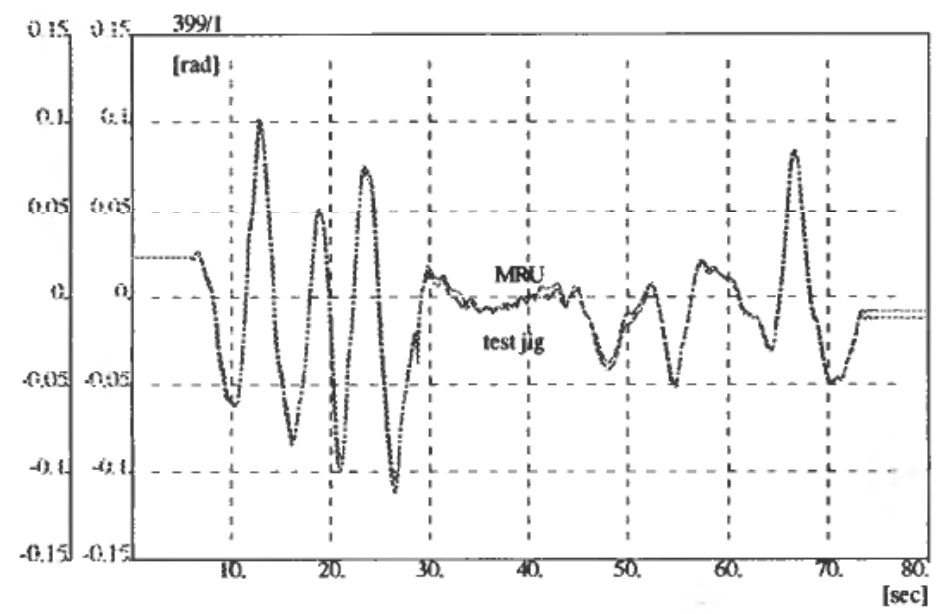

Figure 5. Roll and roll reference recording in test jig. 


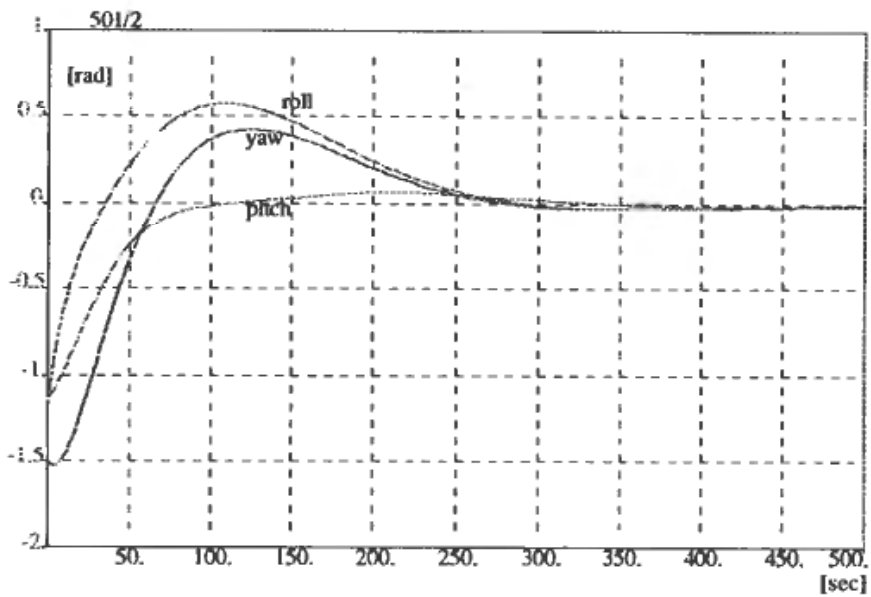

Figure 6. Response in self alignment in roll, pitch, yaw.

\section{Conclusions}

Analysis and experimental results show that an attitude measurement system based on Coriolis force vibratory gyros can be useful for maritime control applications like fast craft roll/pitch control and for enhancement of autopilots. The unit needs external information on speed to provide rated accuracy during turning at high speed.

\section{REFERENCES}

BorTz, J. (1973). A New Mathematical Formulation for Strapdown Inertial Navigation, IEEE Transactions on Aerospace and Electronic Systems, Vol. AES 7, No. 1, Jan.

BuRDESS, J. S. (1986). The dynamics of a thin piezoelectric cylinder gyroscope. Proc. Inst. Mech. Eng., Vol. 200, pp. 271-280.

Fox, C. H. J. (1988). Vibrating Cylinder Rate Gyro, Theory of Operation and Error Analysis, DGON Symposium on Gyro Technology, Stuttgart FRG.

Fox, C. H. J. (1994). Analysis and Control of Imperfection Effects in Vibratory Gyros DGON Symposium on Gyro Technology, Stuttgart FRG.

IGNAGNI, M. B. (1990). Optimal Strapdown Attitude Integration Algorithms, J. Guidance, Vol. 13, No. 2, March/April.

Miller, Robin B. (1983). A New Strapdown Attitude Algorithm, J. Guidance, Vol. 6, No. 4, July/Aug.

Press, William H. (1988) Numerical Recipies in C, The art of scientific Computing, Cambridge University Press. 